\title{
Postoperative Adjuvant Radiochemotherapy for Patients with Stage III or IV Gastric Cancer
}

\author{
Maria Grazia Calvanese ${ }^{1}$, Roberto Manzo $^{2}$, Michele Orditura ${ }^{3}$, Paola Murino ${ }^{2}$, Fabrizio Camma- \\ rota $^{2}$, Rossella Di Franco ${ }^{1}$, Sara Falivene ${ }^{1}$, Anna Morra ${ }^{5}$, Paolo Muto ${ }^{4}$, Vincenzo Ravo*,2 \\ ${ }^{1}$ Dipartimento Diagnostica per Immagini e Radioterapia Seconda Università di Napoli, Italy \\ ${ }^{2}$ UOC Radioterapia PO Ascalesi ASLNA1 Napoli, Italy \\ ${ }^{3}$ Divisione di Oncologia Medica Seconda Università di Napoli, Italy \\ ${ }^{4}$ UOC Radioterapia INT Pascale Napoli, Italy \\ ${ }^{5}$ UOC Radioterapia Istituto Europeo di Oncologia (IEO)Milano, Italy
}

\begin{abstract}
Background and Purpose: It is known that radiotherapy or chemotherapy alone don't represent a standard of care as adjuvant treatment for patients with advanced gastric cancer that underwent surgical resection. The purpose in the approach of this cancer is to find an adjuvant treatment that can affect overall survival. Phase 2 studies and randomized trials suggest that a multimodal approach with chemo radiotherapy (CT-RT) can improve overall survival. We analyze the feasibility and toxic effects of chemo radiotherapy (CT-RT) as a post surgical adjuvant treatment in a cohort of patients with high risk gastric cancer.

Methods: We enrolled 48 patients with advanced gastric cancer (Stage III and IV, M0). These patients were submitted to surgical resection and all of them, within 6 weeks, underwent adjuvant chemotherapy with FOLFOX-4 (ie, a combination of folinic acid, fluorouracil, and oxaliplatin) for 8 cycles and concomitant radiotherapy (45 Gy in 25 daily fractions over 5 weeks). Radiotherapy started after the first 2 cycles of FOLFOX-4. Chemotherapy schedule was reduced by $25 \%$ during the period of the contemporary radiotherapy treatment.

Results: All patients except one ended the combined adjuvant treatment. We observed severe hematologic adverse effects only in less than $10 \%$ of patients (4 patients); regarding gastrointestinal toxic effects they occurred in $33 \%$ of patients and specifically we noted G1-G3 grade toxicity and no G4 toxicity . Disease-free and overall survival at 1, 2, and 3 years was superior to in untreated patients. One to 3-years Median disease-free and overall survival rates were 27 months and 15 months respectively.
\end{abstract}

Conclusions: A combined trial with chemo radiotherapy (CT-RT ) as adjuvant treatment represents an effective approach for patients with resected advanced gastric cancer.

Keywords: Chemotherapy, Radiotherapy, Gastric cancer.

\section{INTRODUCTION}

Gastric Cancer is the second leading cause of cancer death worldwide, although its incidence is decreasing [1]. Complete surgical resection remains the only curative treatment option for patients affected by a gastric cancer. Even after complete surgical resection with negative margins (i.e. R0 resection) plus D1 or D2 lymphoadenectomy (i.e. systematic lymphoadenectomy of compartments 1 e 2) many patients, particularly those with stage III and IV (with M0 disease) will eventually relapse [2]. Studies have revealed a high rate of distant failure and also of loco regional relapses even after R0 resection [3,4]. Long-term survival is only

*Address correspondence to this author at the UOC Radioterapia PO Ascalesi Via Egiziaca a Forcella 31, 80131 Napoli-Italy; Tel: 081.2542177/2137/2185; Fax: 081.5630660;

E-mail: enzoravo@libero.it achieved in $8 \%-40 \%$ of patients with loco regionally advanced disease, which makes the evaluation of an adjuvant or neoadjuvant treatment option a priority for these patients [5]. According to the results of the Magic (Medical Research Council Adjuvant Gastric Infusional Chemotherapy) trial [6] the common approach for resectable gastric cancers is, in Europe, constituted by a perioperative chemotherapy. Postoperative fluorouracile-based CT-RT is, instead, the recommended treatment for PT3 and N+ gastric cancers in the United States [7]. In our study obtained on selected patients (stages III or IV, Mo) we want to demonstrate the tolerance and the efficacy of post-operative chemo radiotherapy (CTRT ) as an adjuvant treatment.

\section{MATERIALS AND METHODS}

Between January 2006 and June 2010 patients, a cohort of forty-eight patients, were enrolled. According to our in- 
clusion criteria we selected patients older than 18 years and younger than 75 years, all of them underwent a R0 surgical resection that is a complete removal of all macroscopic tumor masses and absence of microscopic residual tumor, negative margins, lymphoadenectomy extended beyond involved nodes with negative more distant lymph nodes ( D2 lymphodenectomy ). Anyone of them had an histologically confirmed diagnosis of gastric cancer. Patients were submitted to specific examinations to prove their adequate bone marrow, hepatic and renal function. Performance-Status was of 1 or less. Baseline characteristics of the enrolled population are depicted in Table 1.

Median age was 54 (34-75), 34 patients were men and 17 women; most of them had a performance status of 0 . The

Table 1. Patients' Characteristics (48ptz) and their Influence on the 3 Years Survival

\begin{tabular}{|c|c|c|}
\hline & Patients (No) & 3-y Survival (\%) \\
\hline Age,$\leq 51 \mathrm{y} />51 \mathrm{y}$ & $24 / 24$ & $62 / 47$ \\
\hline Sex,M/F & $32 / 16$ & $45 / 80$ \\
\hline Performance Status, 0/1 & $46 / 2$ & $50 / 50$ \\
\hline \multicolumn{3}{|l|}{ Pathologic Grading } \\
\hline G1 & 1 & 100 \\
\hline G2 & 16 & 38 \\
\hline G3 & 31 & 38 \\
\hline Serum CEA level, $\leq 3.5 />3.5 \mathrm{ng} / \mathrm{mL}$ & $39 / 9$ & $60 / 15$ \\
\hline Serum CA19-9 level, $\leq 40 />40 \mathrm{ng} / \mathrm{mL}$ & $38 / 10$ & $67 / 13$ \\
\hline \multicolumn{3}{|l|}{ Site } \\
\hline Antrum & 36 & 65 \\
\hline Fundus & 3 & 100 \\
\hline Corpus & 5 & 0 \\
\hline Cardias & 4 & 60 \\
\hline Size $\leq 4 />4 \mathrm{~cm}^{2}$ & $29 / 19$ & $67 / 29$ \\
\hline \multicolumn{3}{|l|}{ Tumor } \\
\hline $\mathrm{T} 2$ & 2 & 100 \\
\hline $\mathrm{T} 3$ & 42 & 44 \\
\hline $\mathrm{T} 4$ & 4 & 100 \\
\hline \multicolumn{3}{|l|}{ Nodes } \\
\hline N1 & 7 & 80 \\
\hline $\mathrm{N} 2$ & 26 & 39 \\
\hline N3 & 15 & 50 \\
\hline \multicolumn{3}{|l|}{ UICC stage } \\
\hline IIIA & 9 & 100 \\
\hline IIIB & 22 & 53 \\
\hline IV(M0) & 17 & 40 \\
\hline \multicolumn{3}{|l|}{ Bormann classification } \\
\hline I & 1 & 0 \\
\hline II & 12 & 100 \\
\hline III & 22 & 32 \\
\hline IV & 13 & 20 \\
\hline Lauren classification, intestinal/diffuse & $8 / 40$ & $67 / 30$ \\
\hline Ming classification, expansive/infiltrative & $10 / 38$ & $63 / 42$ \\
\hline Vascular-lymphatic invasion, no/yes & $31 / 9$ & $58 / 44$ \\
\hline Resected nodes, $\leq 26 />26$ & $34 / 14$ & $37 / 73$ \\
\hline Metastatic nodes, $\leq 9 / 9$ & $35 / 13$ & $55 / 38$ \\
\hline
\end{tabular}


$64.6 \%$ of the patients had stage III cancer and the $35.4 \%$ had stage IV cancer. Overall, 781 LNs were removed ( mean [SD],26 [10]; range 15-59; median 26 ) and 305 turned out to be metastatic $(\mathrm{LN}+)$.

Chemotherapy-Radiotherapy (CT-RT) schedule consisted on 2 cycles of CT at full dose, followed by contemporary CT-RT (with a CT dose reduced by $25 \%$ ), and then by two more cycles of CT at full dose for a total of eight courses. CT treatment started within 6 weeks of surgery and consisted on oxaliplatin, $85 \mathrm{mg} / \mathrm{m}^{2}$ on day 1 ; folinic acid, 200 $\mathrm{mg} / \mathrm{m}^{2}$ as a 2-hour infusion, followed by bolus fluorouracil, $400 \mathrm{mg} / \mathrm{m}^{2}$; and a 22-hour infusion of fluorouracil, 600 $\mathrm{mg} / \mathrm{m}^{2}$ on days 1 and 2 every 2 weeks (FOLFOX-4). CT-RT treatment started two weeks after the second cycle of Folofox-4. Totally 45 Gy were delivered in 25 fractions, five days per week, with a 3D conformal technique. Computed tomography-based 3-dimensional RT planning was previously performed and radiotherapy was delivered with 18-MV photons in all the patients, generally by means of a 3-field technique.

The Clinical Target Volume (CTV) was defined using preoperative computed tomographs, endoscopic findings, surgical clips, and all other available information and included the gastric bed, the draining LNs as described in the Intergroup 0116 study [8,9], the anastomotic region, and a safety margin around the former tumor involving all mucosal cavity walls for at least $3 \mathrm{~cm}$. The PTV (Planning Target Volume) was calculated with an expansion of $1 \mathrm{~cm}$ over the CTV. So the 45 Gy were delivered in 25 fractions, to the tumor bed, anastomoses and stumps, and regional lymphatics. The design of the radiation treatment fields for postoperative treatment was individualized according to tumor stage and location in the stomach and the type of surgery performed. Lymph node stations included in the radiation fields were perigastric, celiac, splenic hilar, suprapancreatic, porta hepatis, pancreaticoduodenal, and local paraaortic nodes. For proximal lesions involving the gastroesophageal junction, the paraesophageal nodes were also included in the radiation fields. In patients with tumors confined to the proximal third of the stomach or gastroesophageal junction with limited lymphatic invasion (fewer than three nodes positive) treatment of the pancreaticoduodenal nodes and porta hepatis nodes was omitted. Similarly, treatment of the splenic hilar nodes was omitted in patients with tumors of the antrum/lower third of the stomach with limited lymphatic invasion. Clinical target volumes (CTV) and dose-limiting normal tissues for radiotherapy were defined on axial CT slices according to detailed and illustrated CTV delineation guidelines. These guidelines were produced thanks to a multimodal approach between oncologists, radiation oncologists, gastric surgeons, and diagnostic radiologists, and they comprise illustrative CT scans and diagrams that are designed to show practical examples of the CTV contouring process. Dose-volume histograms (DVHs) were recorded for the kidneys, liver and spinal cord in all patients. Baseline hepatic and renal function were evaluated prior to starting chemotherapy and also radiotherapy. During radiotherapy the Folfox- 4 was contemporary applied biweekly at a reduced dose of $25 \%$.

Concerning Chemotherapy (CT) treatment toxic effects were determinate before starting and at each 2-week cycle using the National Cancer Institute Common Toxicity Criteria [10]. Treatment delays and dose modifications were based on the results of a complete hematologic evaluation performed on the day of the planned treatment. When thrombocytopenia or neutropenia grade $(\mathrm{G}>2)$ or other significant non hematologic toxic effects developed, CT recycle was delayed for up to 2 weeks. The fluorouracil dose was reduced in cases of severe Gastro-intestinal toxicity (G3 - 15 patients totally).

During the RT-CT treatment patients were submitted to clinical examination once a week to assess the effect and the toxicity of the treatment. Hematologic parameters were evaluated each 7-10 days. Patient's weight was acquired before starting RT treatment, at half of the treatment and at the end of the treatment. A specific attention was directed to patient nutrition. Most of the patients had no severe symptoms and or major problems in feeding. Enteral or parenteral nutrition was added in seven patients when inadequate intake of energy caused weight loss or body mass index modification.

The primary study end points were determination of toxic effects and the safety profile of the treatment. Secondary end points included DFS and OS rates, calculated from the time of surgery to evidence of relapse or the date of the last evaluation and death, respectively. No patient was lost to follow-up.

\section{RESULTS}

All patients, except one, completed the treatment that was well tolerated. Common adverse effects were gastrointestinal such as nausea, vomiting, abdominal pain, and dyspepsia that were recorded respectively in 35 patients ( $74 \%$ ), 18 patients ( $38 \%), 11$ patients $(24 \%)$ and 8 patients $(17 \%)$. We didn't record any G4 gastrointestinal toxic effect. Biochemistry parameters did not have significant variations, only two patients needed growth factors and only one patient needed a nutritional supplementation. No treatment-related deaths were reported. Toxic effects and their recurrence are resumed in Table 2.

During the first evaluation period of this study (i.e in the period including the start of chemotherapy treatment, the concomitant chemo-radiotherapy treatment and the first 19 months of Follow-UP) 10 patients (34\%) died for reasons linked to the disease and cancer recurrence was observed in 16 patients $(55 \%)$. Eighty percent of cancer recurrences occurred in the first 18 months, and no cancer relapse was observed after 2 years. Median disease-free and overall survival rates were $35 \%$ and $60 \%$ respectively. One-to 3 -year OS was $85.0 \%, 62.6 \%$, and $50.1 \%$, respectively. Mean SD survival time was 27 months. One-to 3-year DFS survival was $79 \%, 35 \%$, and $35 \%$, respectively . Mean and median DFS times were 21 months and 15 months, respectively. We observed a direct connection between male sex, worsening TNM stage, reduction of resected nodes and the presence of high number of metastatic lymph nodes and a worst prognosis that impressed the OS [7]. The peritoneum was the main site of relapse. As a single site of progression, it was shown to be involved in 6 patients $(12,5 \%)$, and 1 patient had simultaneous dissemination to lumboaortic LNs. Ovarian metastases were discovered in 3 women, 1 of whom underwent radi- 
Table 2. Main Toxic Effects Registred in the Study

\begin{tabular}{|lccc|}
\hline Toxic effect & $\begin{array}{c}\text { Grades 1-2, } \\
\text { No.(\%) }\end{array}$ & $\begin{array}{r}\text { Grades 3 } \\
\text { No.(\%) }\end{array}$ & $\begin{array}{c}\text { Grades 4 } \\
\text { No.(\%) }\end{array}$ \\
\hline \hline Hematologic & & & \\
Neutropenya & 23 & 3 & 0 \\
Thrombocytopenia & 13 & 1 & 0 \\
Anemia & 10 & 0 & 0 \\
Febrile neutropenia & 0 & 0 & 0 \\
Gastrointestinal & & & \\
Nausea & 25 & 11 & 0 \\
Vomiting & 15 & 3 & 0 \\
Abdominal Pain & 10 & 1 & 0 \\
Dispepsya & 8 & 0 & 0 \\
Hepatic & 0 & 0 & 0 \\
Neurologic & 16 & 1 & 0 \\
Others & & & 0 \\
Asthenia & 11 & 7 & 0 \\
Allergic & 0 & & \\
\hline
\end{tabular}

cal resection. One patient developed a single-site metastasis in supraclavicular nodes that was treated with RT.One patient developed multiple liver metastases and anastomotic recurrence. Other single sites of disease progression were bone ( 2 patients), pleura (1 patient), and lumboaortic LNs (1 patient). Seven of the progressed patients were treated with CT for advanced disease [7].

We observed a correlation between the preoperative serum CEA levels and the 3 -years survival $(60 \%$ of surviving patients with CEA level $\leq 3,5 \mathrm{ng} / \mathrm{mL}$ vs $15 \%$ with CEA level $>3,5 \mathrm{ng} / \mathrm{mL} ; \mathrm{p}<0,01)$ and with the serum CA-19-9 levels (67\% of surviving patients with CA $19-9 \leq 40 \mathrm{ng} / \mathrm{mL}$ vs $13 \%$ with $>40 \mathrm{ng} / \mathrm{mL} \mathrm{p}=.0003$ ) [7].

Considering the lymph nodes dissection we found that the number of resected nodes $(\leq 26 \mathrm{vs}>26)$ and the number of metastatic lymph nodes ( $\leq 9$ vs $>9$ ) had some influence on the 3 -years survival: $37 \%$ vs $73 \%(p=.08)$ and $55 \%$ vs $38 \%(\mathrm{p}=.22)$ respectively.

\section{DISCUSSION}

Gastric Cancer remains a major cause of cancer-related death in most Western countries. Surgery is the only proven effective therapy, but overall 5-year survival rates remain low after resection.

For patients who underwent surgery, prognosis is determined by a series of factors [24,25] among which depth of invasion [11,12], nodal status [12-14], and metastasis [15-17] are the most important recognized system to predict prognosis.

Recently, adjuvant therapies after gastric carcinoma resection have become a matter of discussion $[9,18]$.The trials in question, however, included a wide variety of different tumor stages, so that adjuvant treatment was administered no matter how advanced the tumor was. Future studies in this field should be stratified on the basis of predicted individual risk to later on select those patients who benefit the most from an adjuvant treatment [19].

Many studies and meta- analysis showed that chemotherapy alone, as adjuvant treatment, does not modify the surgical benefit [20-23], but surely improves the OS and relapsefree survival $[24,25]$.

In the study by the Italian Trials in Medical Oncology (ITMO) Group, Bajetta et al.[20] used a EAP regimen (etoposide,adriamycin and cisplatin) followed by the Machover schedule (fluorouracil and folinic acid) given as adjuvant treatment to patients with poor prognostic factors $(\mathrm{N}+$ or T3/4) finding a drug-related grade $3 / 4$ WHO toxicities that included leukopenia (21\%), nausea and vomiting (14\%), mucositis $(9 \%)$, neutropenia $(3 \%)$ and thrombocytopenia $(2 \%)$. They concluded that there was a limited relative risk reduction in the patients receiving adjuvant therapy $(17 \%$ in DFS and $7 \%$ in OS).

The Italian Group for the Study of Digestive Tract Cancer (GISCAD) using the PELFw regimen, consisting of eight weekly administrations of cisplatin, LV, epidoxorubicin, 5FU and glutathione with the support of filgrastim, or a regimen consisting of six monthly administrations of a 5-day course of 5-FU and LV, daily, 5-FU/LV, found no benefit from an intensive weekly chemotherapy in gastric cancer, with only 19 patients $(9.4 \%)$ that completed the treatment in the PELFw arm and $85(43 \%)$ patients that completed the treatment in the 5-FU/LV arm [21].

In the GOIM 9602 Study De Vita et al. [26] in radically resected IB-IIIB gastric cancers patients, with an adjuvant chemotherapy regimen called ELFE (epirubicin, leucovorin, fluorouracil, etoposide) didn't find any improvement in OS over surgery alone.

The role of RT alone as adjuvant treatment was reported in a randomized trial [27] in which 145 patients received surgery alone, 138 were administered postoperative CT, and 153 were given postoperative RT. No survival differences were reported, but RT offered an advantage in terms of reduction in local recurrence $(27 \%$ with surgery alone vs $10 \%$ with surgery and RT). Forty percent of patients had gross or microscopic residual disease after surgery, and $24 \%$ of patients initially enrolled in the RT arm finally did not receive any treatment with radiation. In fact 19 of 153 patients enrolled to be submitted to post-operative RT didn't begin the treatment with radiations due to death or bad Performance Status; 13 patients refused to be submitted to RT treatment and, moreover, 4 of 153 patients didn't satisfied the entry criteria to start treatment.

So it was concluded that a feasible solution can be founded in a well balanced combination of CT plus RT.

The largest trial evaluating the role of CT-RT as adjuvant treatment was the US Intergroup 0116[9] In this study, 556 patients with resected adenocarcinoma of the stomach or gastroesophageal junction were randomized to receive surgery alone or surgery plus postoperative CT-RT. Survival at 3 years was $50 \%$ vs $40 \%$ in favor of postoperatively treated patients. After 5 years of follow-up, OS was shown to improve by $11.6 \%(28.4 \%$ vs $40 \% ; P<.001)$ and relapse-free survival to increase from $25 \%$ to $31 \%$, both in favor of pa- 
tients treated with postoperative CT-RT as opposed to surgery alone. Loco regional relapse was shown to decrease from $29 \%$ to $19 \%$, CT-RT arm vs surgery alone. However, toxic effects were significantly higher with CT-RT, whereas treatment-related mortality was acceptable $(1 \%$ in the CT-RT arm vs $0 \%$ in the surgery alone arm) [9].

Given these data however, there remain concerns regarding the toxicity of this combined treatment, the optimal chemotherapy regimen and the optimal method of radiotherapy delivery in patients treated previously with surgery.

In our study the aim is to demonstrate that an adequate adjuvant treatment is important for patient's survival and to avoid relapses. RT treatment associated to CT helps the local control of the disease. It's known that Folfox-4 is not the more common regimen used in gastric cancer. Post-operative fluorouracil-based CT represent indeed the standard of care for pT3 and N+ Gastric Cancer in the United States [9], moreover it was recently demonstrated by several phase 2 studies that the bolus of fluorouracil can be substituted with a continuous infusion of fluorouracil and the addition of cisplatin or paclitaxel and this schedule seems to be much more tolerated $[2,28]$. FOLFOX-4 schedule, including continuous fluorouracil infusion and oxaliplatin, used at full dose after surgery and at reduced dose during concomitant RT, can be considered the most active, and recently widely investigated, CT regimen for Gastric Cancer treatment [26]. Therefore his benefit is mainly evident only after adequate surgery. It is also true that this schedule may cause side effects such as stomatitis, nausea, vomiting, and also neurological toxicity but all these effects are treatable with common drugs. Moreover we didn't have evidence of G4 toxicity. Our experience didn't show the occurrence of particularly side effects RTrelated. The most common are nausea, dyspepsia and vomiting, but it must be said that these symptoms are generally amplified by the concomitant $\mathrm{CT}$ treatment.

We observed Grade 2 neutropenia in $23 \%$ of patients and Grade 3 in 3\%. Gastrointestinal toxicity evidenced a Grade 2 nausea in $25 \%$ of patients and Grade 3 in $11 \%$, Grade 2 vomiting in $15 \%$ and Grade 3 in $3 \%$. Moreover we found Grade 2 Neurologic side-effects in $16 \%$ (Grade 3 in $1 \%$ ).

We also observed a correlation between the preoperative serum CEA levels and the 3 -years survival $(60 \%$ of surviving patients with CEA level $\leq 3,5 \mathrm{ng} / \mathrm{mL}$ vs $15 \%$ with CEA level $>3,5 \mathrm{ng} / \mathrm{mL} ; \mathrm{p}<0,01)$ and with the serum CA-19-9 levels ( $67 \%$ of surviving patients with CA19-9 $\leq 40 \mathrm{ng} / \mathrm{mL}$ vs $13 \%$ with $>40 \mathrm{ng} / \mathrm{mL} \mathrm{p}=.0003)^{7}$.

Considering the lymph nodes dissection we found that the number of resected nodes $(\leq 26 \mathrm{vs}>26)$ and the number of metastatic lymph nodes ( $\leq 9$ vs $>9$ ) had some influence on the 3-years survival: $37 \%$ vs $73 \%(p=.08)$ and $55 \%$ vs $38 \%(\mathrm{p}=.22)$ respectively. Also the presence of advanced Bormann grade was significantly associated with a worse DFS rate 7 .

Patients underwent first follow-up 3-4 months after RT treatment. No significant symptoms related to previous RT were referred, late gastrointestinal and hematologic toxicity were G0; nutrition was almost regular and body weight increased. Mean (SD) Follow-Up was 19 months (range 5-36 months).
Given that the CT regimen used in our study is not considered the more active in gastric cancer; we can conclude that the final therapeutic benefit is mainly evident only if adequate surgery can be performed.

As regards radiotherapy, a 3D-conformal therapy can be still considered the "gold standard". Anyway a 3D planning with the respect of the dose-constraints as recently described in an AIRO - Associazione Italiana di Radioterapia Oncologica - publication [29] for liver, kidneys and spinal cord, is of fundamental importance.

In conclusion, we can say that adjuvant CT-RT treatment with FOLFOX-4 and 25 daily fractions of 3D conformal radiotherapy at 1.8 Gy per fraction, totally $45 \mathrm{~Gy}$, were shown to be well tolerated and safe in fit patients with locally advanced gastric cancer after potentially curative surgery.

\section{ACKNOWLEDGEMENTS}

None declared.

\section{COMPETING INTERESTS}

All authors disclose any financial and personal relationships with other people or organizations that could inappropriately influence their work.

\section{REFERENCES}

[1] Sasako M, Sano T, Yamamoto S, et al. D2 lymphadenectomy alone or with para-aortic nodal dissection for gastric cancer. N Engl J Med 2008; 359(5): 453-62.

[2] Kollmannsberger C, Budach W, Stahl M, et al. Adjuvant chemoradiation using 5-fluorouracil/folinic acid/cisplatin with or without paclitaxel and radiation in patients with completely resected highrisk gastric cancer: two cooperative phase II studies of the AIO/ARO/ACO. Ann Oncol 2005; 16(8): 1326-33.

[3] Gunderson LL. Gastric cancer patterns of relapse after surgical resection. Semin Radiat Oncol 2002; 12: 150-61.

[4] Landry J, Tepper JE, Wood WC. Patterns of failure following curative resection of gastric carcinoma. Int $\mathrm{J}$ Radiat Oncol Biol Phys 1990; 19: 1357-62.

[5] Hindah SA, Phillips JL, Menck HR. The National Cancer Data Base report on poor survival U.S. gastric carcinoma patients treated with gastrectomy : fifth edition American joint committee on cancer staging, proximal disease, and the "different disease" hypothesis. Cancer 2000; 88(4): 921-32.

[6] Chua YJ, Cunningham D. The UK NCRI MAGIC trial of perioperative chemotherapy in resectable gastric cancer: implications for clinical practice. Ann Surg Oncol 2007; 14(10): 2687-90.

[7] Orditura M, De Vita F, Galizia G, et al. Adjuvant chemoradiotherapy in patients with stage III or IV radically resected gastric cancer. Arch Surg 2010; 145(3): 233-8.

[8] Marchet A, Mocellin S, Ambrosi A, et al. Italian Research Group for Gastric Cancer Study (GIRCG). The prognostic value of N-ratio in patients with gastric cancer: validation in a large, multicenter series. Eur J Surg Oncol 2008; 34(2): 159-65.

[9] Macdonald JS, Smalley SR, Benedetti J, et al. Chemoradiotherapy after surgery compared with surgery alone for adenocarcinoma of the stomach. N Engl JMed 2001; 345(10): 725-30.

[10] NCI toxicity. Available from: http://www.ucdmc.ucdavis.edu/ clinicaltrials/documents/NCI

[11] Siewert JR, Bottcher K, Stein HJ, et al. Relevant prognostic factors in gastric cancer: ten-year results of the German Gastric Cancer Study. Ann Surg 1998; 228: 449-61.

[12] Janunger KG, Hafstrom L, Glimelius B. Chemotherapy in gastric cancer: a review and updated meta-analyses. Eur J Surg 2002; 168(11): 597-608.

[13] Baba H, Korenaga D, Okamura T, et al. Prognostic factors in gastric cancer with serosal invasion: univariate and multivariate analyses. Arch Surg 1989; 124: 1061-4. 
[14] Bozzetti F, Bonfanti G, Morabito A, et al. A multifactorial approach for the prognosis of patients with carcinoma of the stomach after curative resection. Surg Gynecol Obstet 1986; 162: 229-34.

[15] Allgayer H, Heiss MM, Schildberg FW. Prognostic factors in gastric cancer. Br J Surg 1997; 84: 1651-64.

[16] Bottcher K, Becker K, Busch R, et al. Prognostic factors in stomach cancer: results of a uni- and multivariate analysis. Chirurg 1992; 63: 656-61.

[17] Maruyama K. The most important prognostic factors for gastric cancer patients: a study using univariate and multivariate analyses. Scand J Gastroenterol 1987; 22(suppl 133): 63.

[18] Villacampa MM, del Carmen Galan M, Medina MM, et al. Adjuvant chemotherapy for poor prognosis gastric cancer: our experience. Ann Oncol 2000; 11(suppl 4): 71.

[19] Novotny AR, Schuhmacher C, Busch R, Kattan MW, Brennan MF, Siewert J. Predicting individual survival after gastric cancer resection validation of a u.s.-derived nomogram at a single high-volume center in Europe. Ann Surg 2006; 243(1): 74-81.

[20] Bajetta E, Buzzoni R, Mariani L, et al. Adjuvant chemotherapy in gastric cancer: 5 -year results of a randomized study by the Italian Trials in Medical Oncology (ITMO) Group. Ann Oncol 2002; 13(2): 299-307.

[21] Cascinu S, Labianca R, Barone C, et al. Italian group for the study of digestive tract cancer:adjuvant treatment of high-risk, radically resected gastric cancer patients with 5-fluorouracil, leucovorin, cisplatin, and epidoxorubicin in a randomized controlled trial. J Natl Cancer Inst 2007; 99(8): 601-7.

[22] Mari E, Floriani I, Tinazzi A, et al. Efficacy of adjuvant chemotherapy after curative resection for gastric cancer: a meta-analysis of published randomized trials: a study of the GISCAD (Gruppo
Italiano per lo Studio dei Carcinomi dell'Apparato Digerente). Ann Oncol 2000; 11(7): 837-43.

[23] Nitti D, Wils J, Dos Santos JG, et al. EORTC GI Group; ICCG. Randomized phase III trials of adjuvant FAMTX or FEMTX compared with surgery alone in resected gastric cancer: a combined analysis of the EORTC GI Group and the ICCG. Ann Oncol 2006; 17(2): 262-9.

[24] Earle CC, Maroun JA. Adjuvant chemotherapy after curative resection for gastric cancer in non-Asian patients: revisiting a metaanalysis of randomized trials. Eur J Cancer 1999; 35(7): 1059-64.

[25] Bajetta E, Pozzo C, Di Bartolomeo M, Mariani L, Barone C, Nitti D. Significant serviva benefit of adjuvant chemotherapy in gastric cancer: results of individual patient data based meta-analysis of randomized trials. Paper presented at: 10th Italian National Congress of Medical Oncology 2008 October 11-14; Verona, Italy.

[26] De Vita F, Orditura M, Matano E, et al. A phase II study of beweekly oxaliplatin plus infusional 5 -fluorouracil and folinic acid (Folfox-4) as first-line treatment of advanced gastric cancer patients. Br J Cancer 2005; 92(9): 1644-9.

[27] Hallissey MT, Dunn JA, Ward LC, Allum WH. The second British Stomach Cancer Group trial of adjuvant radiotherapy or chemotherapy in respectable gastric cancer: five-year follow-up. Lancet 1994; 343(8909): 1309-12.

[28] Leong T, Michael M, Foo K, et al. Adjuvant and neoadjuvant therapy for gastric cancer using epirubicin/cisplatin/5-fluorouracil (ECF) and alternative regimens before and after chemoradiation. Brit J Cancer 2003; 89: 1433-8.

[29] Giovanna Mantello - Organi a Rischio e constraints di dose. Gruppo Regionale AIRO Emilia Romagna - Marche: 2009.

Received: January 12, 2012

Revised: January 16, 2012

Accepted: January 18, 2012

(C) Calvanese et al.; Licensee Bentham Open.

This is an open access article licensed under the terms of the Creative Commons Attribution Non-Commercial License (http://creativecommons.org/licenses/ by-nc/3.0/) which permits unrestricted, non-commercial use, distribution and reproduction in any medium, provided the work is properly cited. 